TRANSACTIONS OF THE

AMERICAN MATHEMATICAL SOCIETY

Volume 355, Number 9, Pages 3591-3609

S 0002-9947(03)03139-8

Article electronically published on May 15, 2003

\title{
SHARP FOURIER TYPE AND COTYPE WITH RESPECT TO COMPACT SEMISIMPLE LIE GROUPS
}

\author{
JOSÉ GARCÍA-CUERVA, JOSÉ MANUEL MARCO, AND JAVIER PARCET
}

\begin{abstract}
Sharp Fourier type and cotype of Lebesgue spaces and Schatten classes with respect to an arbitrary compact semisimple Lie group are investigated. In the process, a local variant of the Hausdorff-Young inequality on such groups is given.
\end{abstract}

\section{INTRODUCTION}

Let $1 \leq p \leq 2$. An operator space $E$ is said to have Fourier type $p$ with respect to the compact group $G$ if the vector-valued Fourier transform extends to a completely bounded map

$$
\mathcal{F}_{G, E}: L_{E}^{p}(G) \longrightarrow \mathcal{L}_{E}^{p^{\prime}}(\widehat{G}),
$$

where $p^{\prime}=p /(p-1)$ is the exponent conjugate to $p$. That is, a vector-valued Hausdorff-Young inequality of exponent $p$ is satisfied. Similarly, if we replace the operator $\mathcal{F}_{G, E}$ by its inverse, we get the notion of Fourier cotype $p^{\prime}$ of $E$ with respect to $G$. Following the notation of $[8$, we define the constants

$\mathcal{C}_{p}^{1}(E, G)=\left\|\mathcal{F}_{G, E}\right\|_{c b\left(L_{E}^{p}(G), \mathcal{L}_{E}^{p^{\prime}}(\widehat{G})\right)} \quad$ and $\quad \mathcal{C}_{p^{\prime}}^{2}(E, G)=\left\|\mathcal{F}_{G, E}^{-1}\right\|_{c b\left(\mathcal{L}_{E}^{p}(\widehat{G}), L_{E}^{p^{\prime}}(G)\right)}$.

The Fourier type and cotype become stronger conditions on the pair $(E, G)$ as the exponents $p$ and $p^{\prime}$ approach 2. This gives rise to the notions of sharp Fourier type and cotype exponents. The present paper grew out of the project to investigate the sharp Fourier type and cotype of Lebesgue spaces $L^{p}$ and Schatten classes $S^{p}$, and it is a natural continuation of [8]. However, as we shall see below, some other results have appeared in the process which are interesting in their own right.

In Section 1 we recall that the natural candidates for the sharp Fourier type and cotype of $L^{p}$ and $S^{p}$ (where now $\left.1 \leq p \leq \infty\right)$ are $\min \left(p, p^{\prime}\right)$ and $\max \left(p, p^{\prime}\right)$ respectively. To show that this guess is right, one would have to show that for $1 \leq p<q \leq 2$,

(a) $\mathcal{C}_{q}^{1}\left(L^{p}(\Omega), G\right)=\mathcal{C}_{q^{\prime}}^{2}\left(L^{p^{\prime}}(\Omega), G\right)=\infty$,

(b) $\mathcal{C}_{q}^{1}\left(L^{p^{\prime}}(\Omega), G\right)=\mathcal{C}_{q^{\prime}}^{2}\left(L^{p}(\Omega), G\right)=\infty$

with the obvious modifications for the Schatten classes. In Section 2 we make some remarks about $(a)$ and $(b)$. First we show that, to have any chance of getting

Received by the editors March 22, 2002.

2000 Mathematics Subject Classification. Primary 43A77; Secondary 22E46, 46L07.

Key words and phrases. Sharp Fourier type and cotype, Fourier transform, operator space, compact semisimple Lie group, central function, local Hausdorff-Young inequality.

Research supported in part by the European Commission via the TMR Network "Harmonic Analysis" and by Project BFM 2001/0189, Spain. 
positive answers to these questions, we have to require that the group $G$ not be finite and the operator spaces $L^{p}$ and $S^{p}$ be infinite-dimensional. Then, under such assumptions, one can easily get the following inequality:

$$
\mathcal{C}_{q}^{1}\left(L^{p}(\Omega), G\right) \geq \limsup _{n \rightarrow \infty} \mathcal{C}_{q}^{1}\left(l^{p}(n), G\right)
$$

and the analog for $L^{p^{\prime}}(\Omega)$. Therefore, the growth of $\mathcal{C}_{q}^{1}\left(l^{p}(n), G\right)$ and $\mathcal{C}_{q}^{1}\left(l^{p^{\prime}}(n), G\right)$ provides a possible way to obtain $(a)$ and $(b)$. In the last part of Section 2 we analyze the vector-valued Lebesgue spaces and Schatten classes.

The growth of $\mathcal{C}_{q}^{1}\left(l^{p}(n), G\right)$ is investigated in Section 3 , To be precise, if $G$ stands for a compact semisimple Lie group and $1 \leq p<q \leq 2$, then there exists a constant $0 \leq \mathcal{K}(G, q) \leq 1$ such that $\mathcal{C}_{q}^{1}\left(l^{p}(n), G\right) \geq \mathcal{K}(G, q) n^{1 / p-1 / q}$ for all $n \geq 1$. If one is able to show that $\mathcal{K}(G, q)>0$, this result gives $(a)$. Moreover, we would obtain optimal growth, since $\mathcal{C}_{q}^{1}\left(l^{p}(n), G\right) \leq n^{1 / p-1 / q}$ for any compact group. We shall see that

$$
\mathcal{K}(G, q)=\inf _{n \geq 1} \sup \left\{\frac{\|\widehat{f}\|_{\mathcal{L}^{q^{\prime}}(\widehat{G})}}{\|f\|_{L^{q}(G)}}: \quad f \quad \text { central, } \quad f \in L^{q}(G), \quad \operatorname{supp}(f) \subset \mathcal{U}_{n}\right\}
$$

where $\left\{\mathcal{U}_{n}: n \geq 1\right\}$ is a basis of neighborhoods of $\mathbf{1}$, the identity element of $G$. The Hausdorff-Young inequality on compact groups yields $\mathcal{K}(G, q) \leq 1$. The interesting point lies in the inequality $\mathcal{K}(G, q)>0$, which constitutes a local variant of the Hausdorff-Young inequality on $G$ with exponent $q$.

Sections 4 and 5] are completely devoted to the proof of this local inequality. In the abelian setting, the particular case $G=\mathbb{T}$ was explored by Andersson in 1 . The basic idea is to consider a function $f: \mathbb{T} \rightarrow \mathbb{C}$ as a complex-valued function on $\mathbb{R}$ supported in $[-1 / 2,1 / 2)$. Then, by expressing the norm of $\widehat{f}$ on $L^{q^{\prime}}(\mathbb{R})$ as a Riemann sum, one obtains

$$
\frac{\|\widehat{f}\|_{L^{q^{\prime}}(\mathbb{R})}}{\|f\|_{L^{q}(\mathbb{R})}}=\lim _{k \rightarrow \infty} \frac{\left\|\widehat{\varphi}_{k}\right\|_{L^{q^{\prime}}(\mathbb{T})}}{\left\|\varphi_{k}\right\|_{L^{q}(\mathbb{T})}}
$$

where $\varphi_{k}(t)=k^{1 / q} f(k t)$. This gives $\mathcal{K}(\mathbb{T}, q) \geq \mathcal{B}_{q}$, where $\mathcal{B}_{q}=\sqrt{q^{1 / q} / q^{\prime 1 / q^{\prime}}}$ stands for the Babenko-Beckner constant, see [2] and [3], but in fact the equality holds, as was proved by Sjölin in [15]. We show here that Andersson's argument, suitably modified, is also valid in the context of compact semisimple Lie groups. In Section 4 we summarize the main results of the structure and representation theory of compact semisimple Lie groups that will be used in the process. Then we use these algebraic results to get an expression for the Fourier transform of central functions $f: G \rightarrow \mathbb{C}$ in terms of the Fourier transform $\mathcal{F}_{\mathbf{T}}$ on the maximal torus $\mathbf{T}$ of $G$. This will allow us to work over the maximal torus where we know that Andersson obtained a satisfactory result. However, in the non-commutative setting, the degree $d_{\pi}$ of an irreducible representation $\pi$ does not have to be 1 . We shall see that this becomes a further obstacle, to be treated in Section 5. There we combine some results, such as the Weyl dimension formula, concerning the representation theory of compact semisimple Lie groups with classical harmonic analysis to avoid this difficulty.

On the other hand, if we notice that $\mathcal{C}_{q}^{1}\left(l^{p^{\prime}}(n), G\right)=\mathcal{C}_{q^{\prime}}^{2}\left(l^{p}(n), G\right)$, we can understand the growth of this constant as the dual problem of the growth of $\mathcal{C}_{q}^{1}\left(l^{p}(n), G\right)$ in the sense that we replace the Fourier transform operator $\mathcal{F}_{G, l^{p}(n)}$ by its inverse. 
Therefore, since the dual object is no longer a group (as it is when $G$ is abelian) we do not have a Fourier inversion theorem and we should not expect to reconstruct the proof given in Sections 3 , 4and5 step by step. At the time of this writing, we are not able to solve this problem, and so we pose it as follows:

Problem. Let $G$ be any compact semisimple Lie group and let $1 \leq p<q \leq 2$. Does the estimate $\mathcal{C}_{q}^{1}\left(l^{p^{\prime}}(n), G\right) \geq \mathcal{K}(G, q) n^{1 / p-1 / q}$ hold for some positive constant $\mathcal{K}(G, q)$ depending only on $G$ and $q$ ?

Finally, we point to a non-commutative notion of Rademacher type for operator spaces, see [9]. We think this notion could be helpful in order to study the growth of $\mathcal{C}_{q}^{1}\left(l^{p^{\prime}}(n), G\right)$.

\section{Statement of the Problem}

All throughout this paper some basic notions of operator space theory and noncommutative vector-valued discrete $L^{p}$ spaces will be assumed. The definitions and results about operator spaces that we are using can be found in the book of Effros and Ruan [5, while for the study of our non-commutative $L^{p}$ spaces the reader is referred to [12], where Pisier analyzes them in detail. In any case, all the analytic preliminaries of this paper are summarized in [8], where we study the Fourier type and cotype of an operator space with respect to a compact group. In order to state the problem we want to solve, we begin by recalling the definitions and the main properties of Fourier type and cotype.

Let $G$ be a compact topological group endowed with its Haar measure $\mu$ normalized so that $\mu(G)=1$, and let $\pi \in \widehat{G}$ be an irreducible unitary representation of $G$ of degree $d_{\pi}$. Here the symbol $\widehat{G}$ stands for the dual object of $G$. Given an operator space $E$, it was shown in 8 that, by fixing a basis on the representation space of each $\pi \in \widehat{G}$, the Fourier transform operator $\mathcal{F}_{G, E}$ for functions defined on $G$ and with values on $E$ has the form $f \in L_{E}^{1}(G) \longmapsto(\widehat{f}(\pi))_{\pi \in \widehat{G}} \in \mathcal{M}_{E}(\widehat{G})$, where

$$
\widehat{f}(\pi)=\int_{G} f(g) \pi(g)^{\star} d \mu(g) \quad \text { and } \quad \mathcal{M}_{E}(\widehat{G})=\prod_{\pi \in \widehat{G}} M_{d_{\pi}} \otimes E .
$$

Here $M_{n}$ denotes the space of $n \times n$ complex matrices. Let $1 \leq p<\infty$. If $S_{n}^{p}(E)$ stands for the vector-valued Schatten class on $M_{n} \otimes E$, we define the spaces

$$
\begin{aligned}
& \text { - } \mathcal{L}_{E}^{p}(\widehat{G})=\left\{A \in \mathcal{M}_{E}(\widehat{G}):\|A\|_{\mathcal{L}_{E}^{p}(\widehat{G})}=\left(\sum_{\pi \in \widehat{G}} d_{\pi}\left\|A^{\pi}\right\|_{S_{d_{\pi}}^{p}(E)}^{p}\right)^{1 / p}<\infty\right\}, \\
& \text { - } \mathcal{L}_{E}^{\infty}(\widehat{G})=\left\{A \in \mathcal{M}_{E}(\widehat{G}):\|A\|_{\mathcal{L}_{E}^{\infty}(\widehat{G})}=\sup _{\pi \in \widehat{G}}\left\|A^{\pi}\right\|_{S_{d_{\pi}}^{\infty}(E)}<\infty\right\} .
\end{aligned}
$$

We write $\mathcal{L}^{p}(\widehat{G})$ for the case $E=\mathbb{C}$. Finally, let $1 \leq p \leq 2$. Then by the HausdorffYoung inequality on compact groups (see [8] or Kunze's paper [10]) it is not difficult to check that $\mathcal{F}_{G, E}\left(L^{p}(G) \otimes E\right) \subset \mathcal{L}^{p^{\prime}}(\widehat{G}) \otimes E$ and $\mathcal{F}_{G, E}^{-1}\left(\mathcal{L}^{p}(\widehat{G}) \otimes E\right) \subset L^{p^{\prime}}(G) \otimes E$. This motivates the following definitions.

Definition 1.1. Let $1 \leq p \leq 2$ and let $p^{\prime}$ denote its conjugate exponent. The operator space $E$ has Fourier type $p$ with respect to the compact group $G$ if the Fourier transform operator

$$
\mathcal{F}_{G, E}: L^{p}(G) \otimes E \rightarrow \mathcal{L}^{p^{\prime}}(\widehat{G}) \otimes E
$$


can be extended to a completely bounded operator from $L_{E}^{p}(G)$ into $\mathcal{L}_{E}^{p^{\prime}}(\widehat{G})$. In that case, $\mathcal{C}_{p}^{1}(E, G)$ will stand for its $c b$ norm.

Definition 1.2. In the same fashion, the operator space $E$ has Fourier cotype $p^{\prime}$ with respect to the compact group $G$ if the inverse

$$
\mathcal{F}_{G, E}^{-1}: \mathcal{L}^{p}(\widehat{G}) \otimes E \rightarrow L^{p^{\prime}}(G) \otimes E
$$

can be extended to a completely bounded operator from $\mathcal{L}_{E}^{p}(\widehat{G})$ to $L_{E}^{p^{\prime}}(G)$. As before, we shall denote its $c b$ norm by $\mathcal{C}_{p^{\prime}}^{2}(E, G)$.

One of the properties proved in 8 is that every operator space has Fourier type 1 and Fourier cotype $\infty$ with respect to any compact group. In particular, the complex interpolation method for operator spaces (see Pisier's work 11]) provides the following result.

Lemma 1.3. Let $1 \leq p_{1} \leq p_{2} \leq 2$ and assume that $E$ has Fourier type $p_{2}$ with respect to $G$. Then $E$ has Fourier type $p_{1}$ with respect to $G$. Similarly, Fourier cotype $p_{2}^{\prime}$ of $E$ with respect to $G$ implies Fourier cotype $p_{1}^{\prime}$ of $E$ with respect to $G$.

Therefore, the Fourier type and cotype become stronger conditions on the pair $(E, G)$ as the exponent $p$ (and consequently its conjugate $p^{\prime}$ ) tends to 2 . So Lemma 1.3 gives rise to the following definition.

Definition 1.4. The sharp Fourier type and cotype exponents of an operator space $E$ with respect to the compact group $G$ are defined respectively by

$$
\begin{aligned}
& p_{1}(E, G)=\sup \{p \leq 2: E \text { has Fourier type } p \text { with respect to } G\}, \\
& p_{2}(E, G)=\inf \left\{p^{\prime} \geq 2: E \text { has Fourier cotype } p^{\prime} \text { with respect to } G\right\} .
\end{aligned}
$$

If $E$ has Fourier type $p_{1}(E, G)$ with respect to $G$, we say that $E$ has sharp Fourier type $p_{1}(E, G)$. The sharp Fourier cotype of $E$ is defined analogously.

In order to simplify the statement of the problem we shall need the following lemma (see [8]) which analyzes the Fourier type and cotype of the dual $E^{\star}$ of an operator space $E$ with respect to a compact group $G$.

Lemma 1.5. Let $1 \leq p \leq 2$ and let $p^{\prime}$ be the conjugate exponent of $p$. Then we have the equalities $\mathcal{C}_{p}^{1}\left(E^{\star}, G\right)=\mathcal{C}_{p^{\prime}}^{2}(E, G)$ and $\mathcal{C}_{p^{\prime}}^{2}\left(E^{\star}, G\right)=\mathcal{C}_{p}^{1}(E, G)$.

The problem we want to investigate in this paper is how to find the sharp Fourier type and cotype of Lebesgue spaces and Schatten classes. Concerning these topics, we present here a result given in 8 from which we start out. In what follows $(\Omega, \mathcal{A}, \nu)$ will denote a $\sigma$-finite or regular measure space and $S_{\mathbb{N}}^{p}$ the classical Schatten class over the space of compact operators on $l^{2}$.

Theorem 1.6. If $1 \leq p \leq \infty$, then the spaces $L^{p}(\Omega)$, $S_{n}^{p}$ and $S_{\mathbb{N}}^{p}$ have Fourier type $\min \left(p, p^{\prime}\right)$ and Fourier cotype $\max \left(p, p^{\prime}\right)$. In fact, the vector-valued Fourier transform, or its inverse, is a complete contraction in each of the cases considered.

Therefore, if we consider two exponents $p$ and $q$ such that $1 \leq p<q \leq 2$, we would like to find conditions on $G$ and $\Omega$ under which

(a) $\mathcal{C}_{q}^{1}\left(L^{p}(\Omega), G\right)=\mathcal{C}_{q^{\prime}}^{2}\left(L^{p^{\prime}}(\Omega), G\right)=\infty$,

(b) $\mathcal{C}_{q}^{1}\left(L^{p^{\prime}}(\Omega), G\right)=\mathcal{C}_{q^{\prime}}^{2}\left(L^{p}(\Omega), G\right)=\infty$,

with the obvious modifications for the Schatten classes. 


\section{Some REMARKS ABout the PROBlem}

In this section we shall make some remarks about the problem we have just stated. We begin by showing some necessary conditions that should hold to obtain a positive answer to our question. Second, we wonder about sufficient conditions that we shall work with in the rest of this paper. Finally, we study what happens if we consider vector-valued $L^{p}$ spaces (or Bochner-Lebesgue spaces) and vectorvalued Schatten classes.

2.1. Necessary conditions. The first necessary condition that we shall present is on the compact group $G$. We have to exclude finite groups from our treatment, since, as we shall see immediately, every operator space $E$ has sharp Fourier type and cotype 2 with respect to any finite group. Anyway, the next result is a bit more accurate.

Proposition 2.1. For a finite group $G$, every operator space $E$ satisfies the estimates $\mathcal{C}_{p}^{1}(E, G), \mathcal{C}_{p^{\prime}}^{2}(E, G) \leq|G|^{1 / p^{\prime}}$ for $1 \leq p \leq 2$.

Proof. Let us assume that $\mathcal{C}_{2}^{1}(E, G) \leq|G|^{1 / 2}$ for every operator space $E$; then we have $\mathcal{C}_{2}^{2}(E, G)=\mathcal{C}_{2}^{1}\left(E^{\star}, G\right) \leq|G|^{1 / 2}$ by duality. The desired estimates are then obtained by complex interpolation from the equalities $\mathcal{C}_{1}^{1}(E, G)=\mathcal{C}_{\infty}^{2}(E, G)=1$ (proved in [8]) and the case $p=2$. Therefore we focus our attention on the case $p=2$. It suffices to check that for all $m \geq 1$ and any family of functions $\left\{f_{i j}: G \rightarrow\right.$ $E\}_{1 \leq i, j \leq m}$,

$$
\left(\sum_{\pi \in \widehat{G}} d_{\pi}\left\|\left(\widehat{f}_{i j}(\pi)\right)\right\|_{S_{d_{\pi} m}^{2}(E)}^{2}\right)^{1 / 2} \leq|G|^{1 / 2}\left\|\left(f_{i j}\right)\right\|_{S_{m}^{2}\left(L_{E}^{2}(G)\right)} .
$$

But if $G=\left\{g_{1}, g_{2}, \ldots, g_{n}\right\}$, then

$$
\begin{aligned}
\left\|\left(\widehat{f}_{i j}(\pi)\right)\right\|_{S_{d_{\pi} m}^{2}(E)} & =\left\|\left(\frac{1}{n} \sum_{k=1}^{n} f_{i j}\left(g_{k}\right) \pi\left(g_{k}\right)^{\star}\right)\right\|_{S_{d_{\pi} m}^{2}(E)} \\
& \leq \frac{1}{n} \sum_{k=1}^{n}\left\|\pi\left(g_{k}\right)^{\star}\right\|_{S_{d_{\pi}}^{2}}\left\|\left(f_{i j}\left(g_{k}\right)\right)\right\|_{S_{m}^{2}(E)} \\
& \leq d_{\pi}^{1 / 2}\left\|\left(f_{i j}\right)\right\|_{S_{m}^{2}\left(L_{E}^{2}(G)\right)} .
\end{aligned}
$$

Therefore we obtain

$$
\left\|\left(\widehat{f}_{i j}\right)\right\|_{S_{m}^{2}\left(\mathcal{L}_{E}^{2}(\widehat{G})\right)} \leq \sqrt{\sum_{\pi \in \widehat{G}} d_{\pi}^{2}}\left\|\left(f_{i j}\right)\right\|_{S_{m}^{2}\left(L_{E}^{2}(G)\right)},
$$

and, since $\sum_{\pi \in \widehat{G}} d_{\pi}^{2}=|G|$ by the Peter-Weyl theorem, we are done.

Next we show that we cannot work with measure spaces $(\Omega, \mathcal{A}, \nu)$ that are a union of finitely many $\nu$-atoms. Before that we need to define the $c b$ distance between two operator spaces. It is due to Pisier, and it constitutes the analog of the Banach-Mazur distance between two Banach spaces in the context of operator space theory. Given two operator spaces $E_{1}$ and $E_{2}$, we define their $c b$ distance by the relation $d_{c b}\left(E_{1}, E_{2}\right)=\inf \left\{\|u\|_{c b\left(E_{1}, E_{2}\right)}\left\|u^{-1}\right\|_{c b\left(E_{2}, E_{1}\right)}\right\}$ where the infimum runs over all complete isomorphisms $u: E_{1} \rightarrow E_{2}$. The following result (also extracted 
from [8]) relates the Fourier type and cotype of two operator spaces $E_{1}$ and $E_{2}$ to their $c b$ distance.

Lemma 2.2. Let $1 \leq p \leq 2$ and let $E_{1}, E_{2}$ be operator spaces. Then

$$
\mathcal{C}_{p}^{1}\left(E_{2}, G\right) \leq d_{c b}\left(E_{1}, E_{2}\right) \mathcal{C}_{p}^{1}\left(E_{1}, G\right)
$$

and

$$
\mathcal{C}_{p^{\prime}}^{2}\left(E_{2}, G\right) \leq d_{c b}\left(E_{1}, E_{2}\right) \mathcal{C}_{p^{\prime}}^{2}\left(E_{1}, G\right) .
$$

Proposition 2.3. Let $1 \leq p<q \leq 2$ and assume that $(\Omega, \mathcal{A}, \nu)$ is a union of finitely many $\nu$-atoms. Then every compact group $G$ satisfies the following estimates:

$$
\begin{aligned}
\mathcal{C}_{q}^{1}\left(L^{p}(\Omega), G\right) & =\mathcal{C}_{q^{\prime}}^{2}\left(L^{p^{\prime}}(\Omega), G\right) \leq \nu(\Omega)^{1 / p-1 / q}, \\
\mathcal{C}_{q}^{1}\left(L^{p^{\prime}}(\Omega), G\right) & =\mathcal{C}_{q^{\prime}}^{2}\left(L^{p}(\Omega), G\right) \leq \nu(\Omega)^{1 / q^{\prime}-1 / p^{\prime}} .
\end{aligned}
$$

In fact, since $1 / p-1 / q=1 / q^{\prime}-1 / p^{\prime}$, we have the same bound for both $c b$ norms.

Proof. Applying Lemma 2.2 and the last part of Theorem[1.6, we get the estimates $\mathcal{C}_{q}^{1}\left(L^{p}(\Omega), G\right) \leq d_{c b}\left(L^{p}(\Omega), L^{q}(\Omega)\right)$ and $\mathcal{C}_{q}^{1}\left(L^{p^{\prime}}(\Omega), G\right) \leq d_{c b}\left(L^{p^{\prime}}(\Omega), L^{q^{\prime}}(\Omega)\right)$. On the other hand, it is straightforward to check that, for $1 \leq p_{1}<p_{2} \leq \infty$ and such a measure space $(\Omega, \mathcal{A}, \nu)$, we have $d_{c b}\left(L^{p_{1}}(\Omega), L^{p_{2}}(\Omega)\right) \leq \nu(\Omega)^{1 / p_{1}-1 / p_{2}}$.

In other words, we do not allow finite-dimensional Lebesgue spaces. Since the $c b$ distance between two Schatten classes of the same finite dimension is also finite, the arguments used in Proposition 2.3. Theorem 1.6 and Lemma 2.2 are also valid to show that the only Schatten classes that could make Theorem 1.6 sharp are those of infinite dimension.

2.2. Sufficient conditions. The Fourier type and cotype of the subspaces of a given operator space $E$ are bounded above by the respective type and cotype of $E$. The proof of this result is straightforward, see [8].

Lemma 2.4. Let $1 \leq p \leq 2$ and let $F$ be a closed subspace of $E$. Then we have the estimates $\mathcal{C}_{p}^{1}(F, G) \leq \overline{\mathcal{C}}_{p}^{1}(E, G)$ and $\mathcal{C}_{p^{\prime}}^{2}(F, G) \leq \mathcal{C}_{p^{\prime}}^{2}(E, G)$.

After the conditions above, we shall work in the sequel with infinite compact groups and infinite-dimensional Lebesgue spaces and Schatten classes. Since the measure space $(\Omega, \mathcal{A}, \nu)$ is no longer a union of finitely many $\nu$-atoms, we obtain that the $n$-dimensional space $l^{p}(n)$ is a closed subspace of $L^{p}(\Omega)$ for all $n \geq 1$ and any $1 \leq p \leq \infty$. Moreover, recalling that the subspace of diagonal matrices of $S_{n}^{p}$ is completely isomorphic to $l^{p}(n)$, we deduce that the same happens for the Schatten classes $S_{\mathbb{N}}^{p}$. Hence sharpness of Theorem 1.6 will be guaranteed if, for $1 \leq p<q \leq 2$, we have

$\left(a^{\prime}\right) \quad \mathcal{C}_{q}^{1}\left(l^{p}(n), G\right)=\mathcal{C}_{q^{\prime}}^{2}\left(l^{p^{\prime}}(n), G\right) \quad \longrightarrow \quad \infty \quad$ as $n \rightarrow \infty$

$\left(b^{\prime}\right) \quad \mathcal{C}_{q}^{1}\left(l^{p^{\prime}}(n), G\right)=\mathcal{C}_{q^{\prime}}^{2}\left(l^{p}(n), G\right) \quad \longrightarrow \infty \quad$ as $n \rightarrow \infty$.

Therefore our aim from now on will be the study of the growth of the constants $\mathcal{C}_{q}^{1}\left(l^{p}(n), G\right)$ and $\mathcal{C}_{q}^{1}\left(l^{p^{\prime}}(n), G\right)$. The first remark about these constants that we can already make is that both have a common upper bound:

$$
\mathcal{C}_{q}^{1}\left(l^{p}(n), G\right), \quad \mathcal{C}_{q}^{1}\left(l^{p^{\prime}}(n), G\right) \leq n^{1 / p-1 / q} .
$$

This is an obvious consequence of Proposition 2.3 . 
2.3. Vector-valued spaces. Theorem[1.6 was also studied in 8 for vector-valued spaces. Here is the statement of the result obtained there.

Theorem 2.5. Let $1 \leq p \leq \infty$ and let $E$ be an operator space having Fourier type $\min \left(p, p^{\prime}\right)$ (respectively, Fourier cotype $\left.\max \left(p, p^{\prime}\right)\right)$ with respect to $G$. Then the spaces $L_{E}^{p}(\Omega), S_{n}^{p}(E)$ and $S_{\mathbb{N}}^{p}(E)$ have Fourier type $\min \left(p, p^{\prime}\right)$ (respectively, Fourier cotype $\left.\max \left(p, p^{\prime}\right)\right)$ with respect to $G$.

Let $1 \leq p \leq \infty$ and $\min \left(p, p^{\prime}\right)<q \leq 2$. Let $E$ be as in Theorem 2.5. Then Lemma 2.4 gives the following estimates:

$$
\begin{array}{lll}
\mathcal{C}_{q}^{1}\left(L_{E}^{p}(\Omega), G\right) \geq \mathcal{C}_{q}^{1}\left(L^{p}(\Omega), G\right), & \mathcal{C}_{q}^{1}\left(L_{E}^{p}(\Omega), G\right) \geq \mathcal{C}_{q}^{1}(E, G), \\
\mathcal{C}_{q^{\prime}}^{2}\left(L_{E}^{p}(\Omega), G\right) \geq \mathcal{C}_{q^{\prime}}^{2}\left(L^{p}(\Omega), G\right), & \mathcal{C}_{q^{\prime}}^{2}\left(L_{E}^{p}(\Omega), G\right) \geq \mathcal{C}_{q^{\prime}}^{2}(E, G),
\end{array}
$$

with the obvious modifications for the Schatten classes. Hence we have shown that sharp Fourier type or cotype of $L^{p}(\Omega)$ (respectively $S_{\mathbb{N}}^{p}$ ) provides sharp Fourier type or cotype of $L_{E}^{p}(\Omega)$ (respectively $S_{\mathbb{N}}^{p}(E)$ ) with respect to $G$. Also, the same conclusion is obtained assuming sharp Fourier type or cotype of $E$. In particular, the sufficient condition given above also works for vector-valued spaces. Therefore we focus our attention on the growth of the constants $\mathcal{C}_{q}^{1}\left(l^{p}(n), G\right)$ and $\mathcal{C}_{q}^{1}\left(l^{p^{\prime}}(n), G\right)$.

\section{ON The GROWTH OF $\mathcal{C}_{q}^{1}\left(l^{p}(n), G\right)$.}

We shall assume in what follows that $G$ is a compact semisimple Lie group. Semisimplicity is an essential assumption in the arguments that we shall be using. Anyway, for the moment, the only property of such groups that we shall apply is the existence of a maximal torus $\mathbf{T}$ in $G$. The following result gives, in particular, statement $(a)$ in Section 1, with the obvious modifications for Schatten classes, whenever we work with infinite-dimensional operator spaces and compact semisimple Lie groups.

Theorem 3.1. Let $1 \leq p<q \leq 2$ and let $G$ be a compact semisimple Lie group. Then there exists a constant $0<\mathcal{K}(G, q) \leq 1$, depending on $G$ and $q$, such that for all $n \geq 1$,

$$
\mathcal{K}(G, q) n^{1 / p-1 / q} \leq \mathcal{C}_{q}^{1}\left(l^{p}(n), G\right) \leq n^{1 / p-1 / q} .
$$

In particular, we observe that the growth of $\mathcal{C}_{q}^{1}\left(l^{p}(n), G\right)$ is optimal for compact semisimple Lie groups. The proof of this result starts by applying the existence of a maximal torus $\mathbf{T}$ to consider a countable family $\left\{g_{k}: k \geq 1\right\}$ of pairwise commuting elements of $G$; just take $g_{k} \in \mathbf{T}$. For every $n \geq 1$ we take $\mathcal{U}_{n}$ to be a neighborhood of 1 (the identity element of $G$ ) satisfying

$$
g_{j}^{-1} \mathcal{U}_{n} \cap g_{k}^{-1} \mathcal{U}_{n}=\emptyset \quad \text { for } 1 \leq j, k \leq n \text { and } j \neq k .
$$

We recall here that we can always consider a central function $f_{n}$ supported in $\mathcal{U}_{n}$ and belonging to $L^{q}(G)$. For example, take $\mathcal{U}_{n}$ to be invariant under conjugations (see Lemma (5.24) of [6]) and $f_{n}=\mathcal{1}_{\mathcal{U}_{n}}$ where $\mathcal{L}_{\mathcal{U}}$ stands for the characteristic function of $\mathcal{U}$. Henceforth $f_{n}$ will be a central function in $L^{q}(G)$ supported in $\mathcal{U}_{n}$, to be fixed later. Then we define the function $\Phi_{n}: G \rightarrow \mathbb{C}^{n}$ by $\Phi_{n}(g)=$ $\left(f_{n}\left(g_{1} g\right), f_{n}\left(g_{2} g\right), \ldots, f_{n}\left(g_{n} g\right)\right)$. We obviously have the estimate

$$
\mathcal{C}_{q}^{1}\left(l^{p}(n), G\right) \geq \frac{\left\|\widehat{\Phi}_{n}\right\|_{\mathcal{L}_{l p}^{q^{\prime}(n)}(\widehat{G})}}{\left\|\Phi_{n}\right\|_{L_{l p}^{q}(n)}(G)} .
$$


So it suffices to prove that this quotient is bounded below by $\mathcal{K}(G, q) n^{1 / p-1 / q}$. The following lemma will be very helpful for that purpose.

Lemma 3.2. Let $1 \leq p_{1}, p_{2} \leq \infty, \pi \in \widehat{G}$ and $n \geq 1$. Consider the matrix-valued vector $\mathrm{A}_{\pi, n}=\left(\pi\left(g_{1}\right), \pi\left(g_{2}\right), \ldots, \pi\left(g_{n}\right)\right)$. Then

$$
\left\|\mathrm{A}_{\pi, n}\right\|_{l_{S_{d_{\pi}}^{p_{2}}}^{p_{1}}(n)}=\left\|\mathrm{A}_{\pi, n}\right\|_{S_{d_{\pi}}^{p_{2}}\left(l^{p_{1}}(n)\right)}=n^{1 / p_{1}} d_{\pi}^{1 / p_{2}}
$$

Proof. Since $g_{1}, g_{2}, \ldots, g_{n}$ are pairwise commuting, there exists a basis of $\mathbb{C}^{d_{\pi}}$ of common eigenvectors of $\pi\left(g_{1}\right), \pi\left(g_{2}\right), \ldots, \pi\left(g_{n}\right)$. Therefore, in that basis, all these matrices are diagonal:

$$
\pi\left(g_{k}\right)=\left(\begin{array}{ccc}
\theta_{1}^{k} & & \\
& \ddots & \\
& & \theta_{d_{\pi}}^{k}
\end{array}\right) .
$$

Moreover, $\left|\theta_{j}^{k}\right|=1$ for $1 \leq j \leq d_{\pi}$ because of the unitarity of $\pi\left(g_{k}\right)$. Hence, applying the complete isometry (see Corollary (1.3) of [12]) between $l_{E}^{p_{2}}\left(d_{\pi}\right)$ and the subspace of diagonal matrices of $S_{d_{\pi}}^{p_{2}}(E)$, we easily obtain the desired equality.

(i) The value of $\left\|\widehat{\Phi}_{n}\right\|_{\mathcal{L}_{l^{p}(n)}^{q^{\prime}}(\widehat{G})}$. We begin by recalling that, since $f_{n}$ is central,

$$
\widehat{f}_{n}(\pi)=\frac{1}{d_{\pi}} \int_{G} f_{n}(g) \overline{\chi_{\pi}(g)} d \mu(g) 1_{d_{\pi}}=\gamma_{\pi, n} 1_{d_{\pi}}
$$

by Schur's lemma. Here $\chi_{\pi}$ is the irreducible character associated to $\pi$ and $1_{m}$ denotes the identity matrix of order $m \times m$. On the other hand, $f_{n}\left(g_{k} \cdot\right)$ is the translation by $g_{k}$ of $f_{n}$; therefore,

$$
\widehat{\Phi}_{n}(\pi)=\frac{1}{d_{\pi}} \int_{G} f_{n}(g) \overline{\chi_{\pi}(g)} d \mu(g)\left(\pi\left(g_{1}\right), \pi\left(g_{2}\right), \ldots, \pi\left(g_{n}\right)\right)=\gamma_{\pi, n} A_{\pi, n}
$$

So we get, by Lemma 3.2, the following equality:

$$
\begin{aligned}
\left\|\widehat{\Phi}_{n}\right\|_{\mathcal{L}_{l^{\prime}(n)}^{q^{\prime}}(\widehat{G})} & =\left(\sum_{\pi \in \widehat{G}} d_{\pi}\left|\gamma_{\pi, n}\right|^{q^{\prime}}\left\|A_{\pi, n}\right\|_{\left.S_{d_{\pi}}^{q^{\prime}(l p}(n)\right)}^{q^{\prime}}\right)^{1 / q^{\prime}} \\
& =n^{1 / p}\left(\sum_{\pi \in \widehat{G}} d_{\pi}^{2}\left|\gamma_{\pi, n}\right|^{q^{\prime}}\right)^{1 / q^{\prime}}=n^{1 / p}\left\|\widehat{f}_{n}\right\|_{\mathcal{L}^{q^{\prime}}(\widehat{G}) .}
\end{aligned}
$$

(ii) The value of $\left\|\Phi_{n}\right\|_{L_{l^{p}(n)}^{p}(G)}$. We have

$$
\begin{aligned}
\left\|\Phi_{n}\right\|_{L_{l^{p}(n)}^{q}(G)} & =\left(\int_{G}\left(\sum_{k=1}^{n}\left|f_{n}\left(g_{k} g\right)\right|^{p}\right)^{q / p} d \mu(g)\right)^{1 / q} \\
& =\left(\sum_{k=1}^{n}\left\|f_{n}\left(g_{k} \cdot\right)\right\|_{L^{q}(G)}^{q}\right)^{1 / q}=n^{1 / q}\left\|f_{n}\right\|_{L^{q}(G)}
\end{aligned}
$$

since the sets $\left\{g_{k}^{-1} \mathcal{U}_{n}: 1 \leq k \leq n\right\}$ are pairwise disjoint.

In summary, we have obtained that $\mathcal{C}_{q}^{1}\left(l^{p}(n), G\right) \geq \mathcal{K}(G, q, n) n^{1 / p-1 / q}$ where the constant $\mathcal{K}(G, q, n)$ is given by

$$
\mathcal{K}(G, q, n)=\frac{\left\|\widehat{f}_{n}\right\|_{\mathcal{L}^{q^{\prime}}(\widehat{G})}}{\left\|f_{n}\right\|_{L^{q}(G)}}
$$


If we define $\mathcal{K}(G, q)=\inf _{n \geq 1} \mathcal{K}(G, q, n)$, it is obvious that $\mathcal{K}(G, q) \leq 1$ by the Hausdorff-Young inequality on compact groups. Thus it remains to check that $\mathcal{K}(G, q)>0$. For that aim, since we have not fixed $f_{n}$ yet, we need to see that

$$
\inf _{n \geq 1} \sup \left\{\frac{\|\widehat{f}\|_{\mathcal{L}^{q^{\prime}}(\widehat{G})}}{\|f\|_{L^{q}(G)}}: \quad f \text { central, } \quad f \in L^{q}(G), \quad \operatorname{supp}(f) \subset \mathcal{U}_{n}\right\}>0 .
$$

We shall prove this fact in Section 5 where we study the supremum of the Hausdorff-Young quotient for central functions supported in arbitrary small sets. As we shall see immediately, semisimplicity of $G$ will be essential in our proof.

\section{A SIMPle EXPRESSION FOR THE Fourier TRANSFORM OF CENTRAL FUNCTIONS}

In this section we apply some basic results concerning the structure and representation theory of compact semisimple Lie groups to provide a simple expression for the Fourier transform of central functions defined on such groups. These algebraic preliminaries can be found in Simon's book [14] or alternatively in [7], but we summarize the main topics here. Let $G$ be a compact semisimple Lie group and let $\mathfrak{g}$ be its Lie algebra. In what follows we choose once and for all an explicit maximal torus $\mathbf{T}$ in $G$, while $\mathfrak{h}$ will stand for its Lie algebra. That is, $\mathfrak{h}$ is the Cartan subalgebra of $\mathfrak{g}$. The rank of $G$ will be denoted by $r$; in particular, $\mathbf{T} \simeq \mathbb{T}^{r}$ where $\mathbb{T}=\mathbb{R} / \mathbb{Z}$ with its natural group structure. Also, as is customary, we consider the complexification $\mathfrak{g}_{\mathbb{C}}=\mathfrak{g} \oplus i \mathfrak{g}$ (with complex conjugates taken so that $\mathfrak{g}_{\mathbb{R}}=\left\{Z \in \mathfrak{g}_{\mathbb{C}}: Z=\bar{Z}\right\}=i \mathfrak{g}$ and similarly $\left.\mathfrak{h}_{\mathbb{R}}=i \mathfrak{h}\right)$ with the complex inner product $\langle$, $\rangle$ induced by the Killing form. We also recall that the Weyl group $\mathcal{W}_{G}$ associated to $G$ can be seen as a set of $r \times r$ unitary matrices $W$ (isometries on $\mathfrak{h}_{\mathbb{R}}$ ) with integer entries and det $W= \pm 1$. In particular, the set $\mathcal{W}_{G}^{\star}=\left\{W^{t}: W \in \mathcal{W}_{G}\right\}$ becomes a set of isometries on $\mathfrak{h}_{\mathbb{R}}^{\star}$. The symbol $\mathcal{R}$ will stand for the set of roots, and, if we take $H_{0} \in \mathfrak{h}_{\mathbb{R}}$ such that $\alpha\left(H_{0}\right) \neq 0$ for any root $\alpha$, the symbol $\mathcal{R}^{+}=\left\{\alpha \in \mathcal{R}: \alpha\left(H_{0}\right)>0\right\}$ denotes the set of positive roots. Finally, we shall write $\Lambda_{\mathrm{W}}$ and $\Lambda_{\mathrm{DW}}$ for the weight lattice and the set of dominant weights, respectively.

Now that we have fixed some notation, let us consider a central function $f: G \rightarrow$ $\mathbb{C}$ and a dominant weight $\lambda \in \Lambda_{\mathrm{DW}}$. By the dominant weight theorem there exists a unique $\pi_{\lambda} \in \widehat{G}$ associated to $\lambda$ and, since $f$ is central, we can write, by Schur's lemma,

$$
\widehat{f}\left(\pi_{\lambda}\right)=\frac{1}{d_{\lambda}} \int_{G} f(g) \overline{\chi_{\lambda}(g)} d \mu(g) 1_{d_{\lambda}}
$$

where $d_{\lambda}$ is the degree of $\pi_{\lambda}, \chi_{\lambda}$ is the character of $\pi_{\lambda}$ and $1_{m}$ denotes the $m \times m$ identity matrix. We now recall the definition of the functions $A_{\beta}$ appearing in the Weyl character formula. Given $\beta \in \mathfrak{h}_{\mathbb{R}}^{\star}$, we define the functions $\exp _{\beta}: \mathfrak{h}_{\mathbb{R}} \rightarrow \mathbb{C}$ and $A_{\beta}: \mathfrak{h}_{\mathbb{R}} \rightarrow \mathbb{C}$ by the relations

$$
\begin{aligned}
\exp _{\beta}(H) & =e^{2 \pi i\langle\beta, H\rangle}, \\
A_{\beta}(H) & =\sum_{W \in \mathcal{W}_{G}} \operatorname{det} W \exp _{\beta}(W(H)) .
\end{aligned}
$$

The maximal torus $\mathbf{T}$ is isomorphic via the exponential mapping to the quotient space $\mathfrak{h}_{\mathbb{R}} / \mathrm{L}_{\mathrm{W}}$, where $\mathrm{L}_{\mathrm{W}}$ is the set of those $H \in \mathfrak{h}_{\mathbb{R}}$ satisfying $\exp (2 \pi i H)=\mathbf{1}$. 
That is, $\mathrm{L}_{\mathrm{W}}$ is the dual lattice of $\Lambda_{\mathrm{W}}$. Therefore, the functions $\exp _{\beta}$ and $A_{\beta}$ are well-defined functions on $\mathbf{T}$ if and only if $\beta \in \Lambda_{\mathrm{W}}$. As we know, the integral form

$$
\delta=\frac{1}{2} \sum_{\alpha \in \mathcal{R}^{+}} \alpha
$$

is not necessarily a weight, and so the functions $\exp _{\delta}$ and $A_{\delta}$ could be not welldefined on $\mathbf{T}$. To avoid this difficulty we assume for the moment that $G$ is simply connected; this condition on $G$ assures that $\delta \in \Lambda_{\mathrm{W}}$. Hence, applying consecutively the Weyl integration formula and the Weyl character formula, we get

$$
\begin{aligned}
\widehat{f}\left(\pi_{\lambda}\right) & =\frac{1}{d_{\lambda}\left|\mathcal{W}_{G}\right|} \int_{\mathbf{T}} f(t) \overline{\chi_{\lambda}(t)}\left|A_{\delta}(t)\right|^{2} d m(t) 1_{d_{\lambda}} \\
& =\frac{1}{d_{\lambda}\left|\mathcal{W}_{G}\right|} \int_{\mathbf{T}} f(t) A_{\delta}(t) \overline{A_{\lambda+\delta}(t)} d m(t) 1_{d_{\lambda}}
\end{aligned}
$$

where $m$ denotes the Haar measure on $\mathbf{T}$ normalized so that $m(\mathbf{T})=1$. Now, if we write $A_{\lambda+\delta}$ as a linear combination of exponentials, we obtain

$$
\begin{aligned}
\widehat{f}\left(\pi_{\lambda}\right) & =\frac{1}{d_{\lambda}\left|\mathcal{W}_{G}\right|} \sum_{W \in \mathcal{W}_{G}} \operatorname{det} W \int_{\mathbf{T}} f(t) A_{\delta}(t) \exp _{-(\lambda+\delta)}(W(t)) d m(t) 1_{d_{\lambda}} \\
& =\frac{1}{d_{\lambda}} \int_{\mathbf{T}} f(t) A_{\delta}(t) \exp _{-(\lambda+\delta)}(t) d m(t) 1_{d_{\lambda}},
\end{aligned}
$$

since $A_{\delta}(W(t))=\operatorname{det} W A_{\delta}(t)$ and $f(W(t))=f(t)$. We recall that, taking coordinates with respect to the basis $\left\{\omega_{1}, \omega_{2}, \ldots, \omega_{r}\right\}$ of fundamental weights, any weight $\lambda \in \Lambda_{\mathrm{W}}$ has integer coordinates. Therefore, we can understand the last expression as the Fourier transform of $f A_{\delta}$ on the maximal torus $\mathbf{T}$ evaluated at $\lambda+\delta$. Hence we have

$$
\widehat{f}\left(\pi_{\lambda}\right)=\frac{1}{d_{\lambda}} \mathcal{F}_{\mathbf{T}}\left(f A_{\delta}\right)(\lambda+\delta) 1_{d_{\lambda}},
$$

for $f: G \rightarrow \mathbb{C}$ central and $G$ any compact semisimple simply connected Lie group. When $G$ is not simply connected, a more careful approach is needed. We have $W^{t}(\delta) \pm \delta \in \Lambda_{\mathrm{W}}$ for all $W \in \mathcal{W}_{G}$. In particular, we note that

$$
\exp _{ \pm \delta} A_{\lambda+\delta}=\sum_{W \in \mathcal{W}_{G}} \operatorname{det} W \exp _{W^{t}(\lambda+\delta) \pm \delta}
$$

is a well-defined function on $\mathbf{T}$ for all $\lambda \in \Lambda_{\mathrm{DW}}$. This remark allows us to write $\overline{\chi_{\lambda}}\left|A_{\delta}\right|^{2}=\left(\exp _{\delta} \overline{A_{\lambda+\delta}}\right)\left(\exp _{-\delta} A_{\delta}\right)$ as a well-defined function on $\mathbf{T}$. Henceforth, applying again Schur's lemma, the Weyl integration formula and the Weyl character formula, we get

$$
\begin{aligned}
\widehat{f}\left(\pi_{\lambda}\right) & =\frac{1}{d_{\lambda}\left|\mathcal{W}_{G}\right|} \sum_{W \in \mathcal{W}_{G}} \operatorname{det} W \int_{\mathbf{T}} f(t)\left(\exp _{-\delta} A_{\delta}\right)(t) \exp _{\delta-W^{t}(\lambda+\delta)}(t) d m(t) 1_{d_{\lambda}} \\
& =\frac{1}{d_{\lambda}} \int_{\mathbf{T}} f(t)\left(\exp _{-\delta} A_{\delta}\right)(t) \exp _{-\lambda}(t) d m(t) 1_{d_{\lambda}}
\end{aligned}
$$

where the last equality follows from the change of variable $t \mapsto W^{t}(t)$. That is, we have shown that

$$
\widehat{f}\left(\pi_{\lambda}\right)=\frac{1}{d_{\lambda}} \mathcal{F}_{\mathbf{T}}\left(f B_{\delta}\right)(\lambda) 1_{d_{\lambda}}
$$


where $B_{\delta}=\exp _{-\delta} A_{\delta}$. This expression is now valid for any compact semisimple Lie group, and it coincides with (1) for simply connected ones.

\section{A local Variant of the Hausdorff-Young inequality ON COMPACT SEMISIMPLE LIE GROUPS}

As we mentioned in the introduction, this section is devoted to the proof of a local variant of the Hausdorff-Young inequality on compact semisimple Lie groups. We recall that this result provides the relation $\mathcal{K}(G, q)>0$ for $1 \leq q \leq 2$, which we needed in Section 3 .

Theorem 5.1. Let $1 \leq q \leq 2$ and let $G$ be a compact semisimple Lie group. Then there exists a constant $0<\mathcal{K}(G, q) \leq 1$ such that, for any open set $\mathcal{U} \subset G$, we have

$$
\sup \left\{\frac{\|\widehat{f}\|_{\mathcal{L}^{q^{\prime}(\widehat{G})}}}{\|f\|_{L^{q}(G)}}: \quad f \quad \text { central, } \quad f \in L^{q}(G), \quad \operatorname{supp}(f) \subset \mathcal{U}\right\} \geq \mathcal{K}(G, q) .
$$

Since the norms of $\widehat{f}$ and $f$ on $\mathcal{L}^{q^{\prime}}(\widehat{G})$ and $L^{q}(G)$ respectively do not change under translations of $f$, we can assume, without loss of generality, that $\mathcal{U}$ is a neighborhood of $\mathbf{1}$. Before proving Theorem 5.1 we need some auxiliary results. Let us assume that $G$ is simply connected and let $f: G \rightarrow \mathbb{C}$ be a central function. A quick look at relation (11) allows us to write

$$
\widehat{f}\left(\pi_{\lambda}\right)=\frac{1}{d_{\lambda}} \operatorname{det} W \mathcal{F}_{\mathbf{T}}\left(f A_{\delta}\right)\left(W^{t}(\lambda+\delta)\right) 1_{d_{\lambda}}
$$

for all $W \in \mathcal{W}_{G}$. On the other hand, let us denote by $P_{\alpha}$ the hyperplane of $\mathfrak{h}_{\mathbb{R}}^{\star}$ orthogonal to $\alpha$ with respect to the complex inner product given by the Killing form. The infinitesimal Cartan-Stiefel diagram is then given by the expression

$$
\mathrm{P}=\bigcup_{\alpha \in \mathcal{R}} P_{\alpha}
$$

Lemma 5.2. Let $G$ be a compact semisimple simply connected Lie group. Then we have $\left\{W^{t}(\lambda+\delta): W \in \mathcal{W}_{G}, \lambda \in \Lambda_{\mathrm{DW}}\right\}=\Lambda_{\mathrm{W}} \backslash \mathrm{P}$. Moreover, the mapping $(W, \lambda) \in \mathcal{W}_{G} \times \Lambda_{\mathrm{DW}} \mapsto W^{t}(\lambda+\delta) \in \Lambda_{\mathrm{W}} \backslash \mathrm{P}$ is injective.

Proof. Since $G$ is simply connected, we have that $\left\{\lambda+\delta: \lambda \in \Lambda_{\mathrm{DW}}\right\}=\Lambda_{\mathrm{W}} \cap \mathbf{C}^{\text {int }}$. Here $\mathbf{C}$ stands for the fundamental Weyl chamber and $\mathbf{C}^{\text {int }}$ for its interior. Now, since $\mathrm{P}$ and $\Lambda_{\mathrm{W}}$ are invariant under the action of $\mathcal{W}_{G}^{\star}$ and for any Weyl chamber C there exists a unique $W \in \mathcal{W}_{G}$ such that $W^{t}(\mathbf{C})=\mathrm{C}$, we obtain the desired equality. Finally, the injectivity follows from the uniqueness mentioned above.

Proposition 5.3. Let $G$ be a compact semisimple simply connected Lie group and let $f: G \rightarrow \mathbb{C}$ be a central function. Then there exists a constant $\mathcal{A}(G, q)$, depending on $G$ and $q$, such that

$$
\|\widehat{f}\|_{\mathcal{L}^{q^{\prime}}(\widehat{G})}=\mathcal{A}(G, q)\left[\sum_{\lambda \in \Lambda_{\mathrm{W}} \backslash \mathrm{P}} \frac{\left|\mathcal{F}_{\mathbf{T}}\left(f A_{\delta}\right)(\lambda)\right|^{q^{\prime}}}{\prod_{\alpha \in \mathcal{R}^{+}}|\langle\alpha, \lambda\rangle|^{q^{\prime}-2}}\right]^{1 / q^{\prime}} .
$$


Proof. Since $f$ is central and $G$ is simply connected, we can apply (3) to obtain

$$
\begin{aligned}
\|\widehat{f}\|_{\mathcal{L}^{q^{\prime}}(\widehat{G})} & =\left[\sum_{\lambda \in \Lambda_{\mathrm{DW}}} d_{\lambda}\left\|\widehat{f}\left(\pi_{\lambda}\right)\right\|_{S_{d_{\lambda}}^{q^{\prime}}}^{q^{\prime}}\right]^{1 / q^{\prime}} \\
& =\left[\frac{1}{\left|\mathcal{W}_{G}\right|} \sum_{W \in \mathcal{W}_{G}} \sum_{\lambda \in \Lambda_{\mathrm{DW}}} d_{\lambda}\left|\frac{1}{d_{\lambda}} \mathcal{F}_{\mathbf{T}}\left(f A_{\delta}\right)\left(W^{t}(\lambda+\delta)\right)\right|^{q^{\prime}}\left\|1_{d_{\lambda}}\right\|_{S_{d_{\lambda}}^{q^{\prime}}}^{q^{\prime}}\right]^{1 / q^{\prime}} .
\end{aligned}
$$

Moreover, the Weyl dimension formula for $d_{\lambda}$ gives

$$
\|\widehat{f}\|_{\mathcal{L}^{q^{\prime}}(\widehat{G})}=\left[\frac{1}{\left|\mathcal{W}_{G}\right|} \prod_{\alpha \in \mathcal{R}^{+}}|\langle\alpha, \delta\rangle|^{q^{\prime}-2} \sum_{W \in \mathcal{W}_{G}} \sum_{\lambda \in \Lambda_{\mathrm{DW}}} \frac{\left|\mathcal{F}_{\mathbf{T}}\left(f A_{\delta}\right)\left(W^{t}(\lambda+\delta)\right)\right|^{q^{\prime}}}{\prod_{\alpha \in \mathcal{R}^{+}}|\langle\alpha, \lambda+\delta\rangle|^{q^{\prime}-2}}\right]^{1 / q^{\prime}} .
$$

Finally, we observe that

$$
\prod_{\alpha \in \mathcal{R}^{+}}|\langle\alpha, \lambda+\delta\rangle|=\prod_{\alpha \in \mathcal{R}}|\langle W(\alpha), \lambda+\delta\rangle|^{1 / 2}=\prod_{\alpha \in \mathcal{R}^{+}}\left|\left\langle\alpha, W^{t}(\lambda+\delta)\right\rangle\right|,
$$

since any $W \in \mathcal{W}_{G}$ is a permutation of the set of roots. Therefore, by Lemma 5.2 we have

$$
\|\widehat{f}\|_{\mathcal{L}^{q^{\prime}}(\widehat{G})}=\left[\frac{1}{\left|\mathcal{W}_{G}\right|} \prod_{\alpha \in \mathcal{R}^{+}}|\langle\alpha, \delta\rangle|^{q^{\prime}-2} \sum_{\lambda \in \Lambda_{\mathrm{W}} \backslash \mathrm{P}} \frac{\left|\mathcal{F}_{\mathbf{T}}\left(f A_{\delta}\right)(\lambda)\right|^{q^{\prime}}}{\prod_{\alpha \in \mathcal{R}^{+}}|\langle\alpha, \lambda\rangle|^{q^{-}-2}}\right]^{1 / q^{\prime}} .
$$

The proof is completed just by taking

$$
\mathcal{A}(G, q)=\left[\frac{1}{\left|\mathcal{W}_{G}\right|} \prod_{\alpha \in \mathcal{R}^{+}}|\langle\alpha, \delta\rangle|^{q^{\prime}-2}\right]^{1 / q^{\prime}} .
$$

We are now ready to give the proof of Theorem 5.1 for simply connected groups. Let $\left\{H_{1}, H_{2}, \ldots, H_{r}\right\}$ be the predual basis of the fundamental weights; any element of $\mathrm{L}_{\mathrm{W}}$ can be written as a linear combination of $H_{1}, H_{2}, \ldots, H_{r}$ with integer coefficients. Then, since $\mathbf{T} \simeq \mathfrak{h}_{\mathbb{R}} / \mathrm{L}_{W}$, we can regard $\mathbf{T}$ as the subset of $\mathfrak{h}_{\mathbb{R}}$ given by

$$
\mathfrak{T}=\left\{\sum_{k=1}^{r} x_{k} H_{k}:-1 / 2 \leq x_{k}<1 / 2\right\} .
$$

On the other hand, let us fix a bounded central function $f_{0}: G \rightarrow \mathbb{C}$; then $f_{0}$ can be understood as a function on $\mathbf{T}$ invariant under the action of $\mathcal{W}_{G}$. Now, since the Weyl group is generated by a set of reflections in $\mathfrak{h}_{\mathbb{R}}, f_{0}$ can be regarded as a complex-valued function on $\mathfrak{h}_{\mathbb{R}}$, supported in $\mathfrak{T}$ and symmetric under such reflections. Let us recall that $\left\{\omega_{1}, \omega_{2}, \ldots, \omega_{r}\right\}$ stands for the basis of fundamental weights. Let $\tau=1-2 / q^{\prime}$; the way we have interpreted the function $f_{0}$ allows us to define the function

$$
I_{\tau} \widehat{\left(f_{0} A_{\delta}\right)}: \mathfrak{h}_{\mathbb{R}}^{\star} \longrightarrow \mathbb{C}
$$

as

$$
\widehat{I_{\tau}\left(\widehat{f_{0} A_{\delta}}\right)}(\xi)=\frac{1}{\prod_{\alpha \in \mathcal{R}^{+}}|\langle\alpha, \xi\rangle|^{\tau}} \mathcal{F}_{\mathfrak{h}_{\mathbb{R}}}\left(f_{0} A_{\delta}\right)(\xi) \quad \text { where } \quad \xi=\sum_{k=1}^{r} \xi_{k} \omega_{k} .
$$


Remark 5.4. The motivation for the notation is that in a classical group such as $S U(2)$ the function just defined is nothing but the Fourier transform of the fractional integral operator

$$
I_{\tau}(f)(x)=\frac{1}{\Gamma(\tau)} \int_{-\infty}^{x} f(y)(x-y)^{\tau-1} d y
$$

acting on $f_{0} A_{\delta}$. Here lies the main difference with the commutative case (where a Hausdorff-Young inequality of local type has been already investigated, see [1]) since the presence of the degrees $d_{\lambda}$ (as a product in Proposition 5.3 by the Weyl dimension formula) requires the presence of a factor of $\mathcal{F}_{\mathfrak{h}_{\mathbb{R}}}\left(f_{0} A_{\delta}\right)$. This fact does not happen in the commutative case, since $d_{\lambda}=1$ for all $\lambda \in \Lambda_{\mathrm{DW}}$.

Lemma 5.5. Let $G$ be a compact semisimple simply connected Lie group and let $f: G \rightarrow \mathbb{C}$ be a central function. Then $\mathcal{F}_{\mathfrak{h}_{\mathbb{R}}}\left(f A_{\delta}\right)(\xi)=0$ for all $\xi \in \mathrm{P}$.

Proof. If $\xi \in \mathrm{P}$, there exists a root $\alpha$ such that $\xi \in P_{\alpha}$. Let $S_{\alpha}$ be the reflection in $P_{\alpha}$; then $\mathcal{F}_{\mathfrak{h}_{\mathbb{R}}}\left(f A_{\delta}\right)(\xi)=\operatorname{det} S_{\alpha} \mathcal{F}_{\mathfrak{h}_{\mathbb{R}}}\left(f A_{\delta}\right)\left(S_{\alpha}(\xi)\right)=-\mathcal{F}_{\mathfrak{h}_{\mathbb{R}}}\left(f A_{\delta}\right)(\xi)$, since, as we know, $S_{\alpha} \in \mathcal{W}_{G}^{\star}$.

The function $\mathcal{F}_{\mathfrak{h}_{\mathbb{R}}}\left(f_{0} A_{\delta}\right)$ is analytic, since $f_{0} A_{\delta}$ has compact support and, by Lemma 5.5, it vanishes at

$$
\mathrm{P}=\left\{\xi \in \mathfrak{h}_{\mathbb{R}}^{\star}: \prod_{\alpha \in \mathcal{R}^{+}}\langle\alpha, \xi\rangle=0\right\} .
$$

In particular, since $0 \leq \tau<1, \widehat{\left.I_{\tau} \widehat{\left(f_{0} A_{\delta}\right.}\right)}$ is continuous and takes the value 0 on $\mathrm{P}$. Now we write the norm of this function in terms of a Riemann sum:

$$
\left.\| I_{\tau} \widehat{\left(f_{0} A_{\delta}\right.}\right) \|_{L^{q^{\prime}\left(\mathfrak{h}_{\mathbb{R}}^{\star}\right)}}=\lim _{k \rightarrow \infty}\left[\sum_{\lambda \in \Lambda_{\mathrm{W}}} \frac{\mathrm{V}_{G}}{k^{r}} \frac{\left|\mathcal{F}_{\mathfrak{h}_{\mathbb{R}}}\left(f_{0} A_{\delta}\right)\left(k^{-1} \lambda\right)\right|^{q^{\prime}}}{\prod_{\alpha \in \mathcal{R}^{+}}\left|\left\langle\alpha, k^{-1} \lambda\right\rangle\right|^{\tau q^{\prime}}}\right]^{1 / q^{\prime}},
$$

where $\mathrm{V}_{G}$ denotes the volume of a cell of $\Lambda_{\mathrm{W}}$. Moreover, $\phi_{k}(x)=k^{\sigma} f_{0}(k x) A_{\delta}(k x)$ is supported in $\mathfrak{T}$ and the relation $\mathcal{F}_{\mathfrak{h}_{\mathbb{R}}}\left(f_{0} A_{\delta}\right)\left(k^{-1} \lambda\right)=k^{r-\sigma} \mathcal{F}_{\mathbf{T}}\left(\phi_{k}\right)(\lambda)$ is satisfied for all $\lambda \in \Lambda_{\mathrm{W}}$. Taking $\sigma=\tau\left|\mathcal{R}^{+}\right|+r / q$, we obtain

$$
\left.\| I_{\tau} \widehat{\left(f_{0} A_{\delta}\right.}\right) \|_{L^{q^{\prime}}\left(\mathfrak{h}_{\mathbb{R}}^{\star}\right)}=\mathrm{V}_{G}^{1 / q^{\prime}} \lim _{k \rightarrow \infty}\left[\sum_{\lambda \in \Lambda_{\mathrm{W}} \backslash \mathrm{P}} \frac{\left|\mathcal{F}_{\mathbf{T}}\left(\phi_{k}\right)(\lambda)\right|^{q^{\prime}}}{\prod_{\alpha \in \mathcal{R}^{+}}|\langle\alpha, \lambda\rangle|^{\tau q^{\prime}}}\right]^{1 / q^{\prime}}
$$

since we know that for $\lambda \in \mathrm{P}$ we get nothing. Finally, let us define $\varphi_{k}: \mathfrak{h}_{\mathbb{R}} \rightarrow \mathbb{C}$ by the relation $\phi_{k}=\varphi_{k} A_{\delta}$. The function $\varphi_{k}$ satisfies $\varphi_{k}(W(x))=\varphi_{k}(x)$ for all $W \in \mathcal{W}_{G}$ and is supported in $k^{-1} \mathfrak{T}$; hence we can understand $\varphi_{k}$ as a central function on $G$. We can also say that, as a consequence of the well-known relation

$$
A_{\delta}=\exp _{-\delta} \prod_{\alpha \in \mathcal{R}^{+}}\left(\exp _{\alpha}-1\right)
$$

$\varphi_{k}$ has no singularities. Therefore Proposition 5.3 provides the following relation for some constant $\mathcal{B}(G, q)$ depending on $G$ and $q$ :

$$
\left.\| I_{\tau} \widehat{\left(f_{0} A_{\delta}\right.}\right)\left\|_{L^{q^{\prime}}\left(\mathfrak{h}_{\mathbb{R}}^{\star}\right)}=\mathcal{B}(G, q) \lim _{k \rightarrow \infty}\right\| \widehat{\varphi}_{k} \|_{\mathcal{L}^{q^{\prime}}(\widehat{G})} .
$$


On the other hand, since $\varphi_{k}$ can be seen as a central function on $G$, we can estimate the norm of $\varphi_{k}$ on $L^{q}(G)$. By the Weyl integration formula we get

$$
\begin{aligned}
\left\|\varphi_{k}\right\|_{L^{q}(G)} & =\left[\frac{1}{\left|\mathcal{W}_{G}\right|} \int_{\mathbf{T}}\left|\varphi_{k} A_{\delta}(t)\right|^{q}\left|A_{\delta}(t)\right|^{2-q} d m(t)\right]^{1 / q} \\
& =\left[\frac{k^{\sigma q}}{\left|\mathcal{W}_{G}\right|} \int_{\mathfrak{T}}\left|f_{0} A_{\delta}(k x)\right|^{q}\left|A_{\delta}(x)\right|^{2-q} d x\right]^{1 / q} \\
& \leq\left[\frac{(2 \pi)^{(2-q)\left|\mathcal{R}^{+}\right|}}{\left|\mathcal{W}_{G}\right|} k^{\sigma q} \int_{\mathfrak{T}^{\sigma}}\left|f_{0} A_{\delta}(k x)\right|^{q} \prod_{\alpha \in \mathcal{R}^{+}}|\alpha(x)|^{2-q} d x\right]^{1 / q},
\end{aligned}
$$

where the last inequality follows from (4). Now, under the change of variable $y=k x$ and taking $\mathcal{C}(G, q)=(2 \pi)^{\tau \mid \mathcal{R}^{+}}\left|\mathcal{W}_{G}\right|^{-1 / q}$, we obtain

$$
\left\|\varphi_{k}\right\|_{L^{q}(G)} \leq \mathcal{C}(G, q) k^{\sigma-\tau\left|\mathcal{R}^{+}\right|-r / q}\left(\int_{\mathfrak{T}^{\prime}}\left|f_{0} A_{\delta}(y)\right|^{q} \prod_{\alpha \in \mathcal{R}^{+}}|\alpha(y)|^{\tau q} d y\right)^{1 / q} .
$$

Recall that $\operatorname{supp}\left(f_{0} A_{\delta}\right) \subset \mathfrak{T}$; therefore the integral over $k \mathfrak{T}$ (the domain of integration after the change of variable) reduces to the same integral over $\mathfrak{T}$. But $\sigma-\tau\left|\mathcal{R}^{+}\right|-r / q=0$, and the product inside the integral is bounded over $\mathfrak{T}$, say by $\mathrm{M}_{G}$. Then we can write

$$
\left\|\varphi_{k}\right\|_{L^{q}(G)} \leq \mathcal{C}(G, q) \mathrm{M}_{G}\left\|f_{0} A_{\delta}\right\|_{L^{q}\left(\mathfrak{h}_{\mathbb{R}}\right)} .
$$

In summary, by (5) and (6), we know there exists a constant $\mathcal{D}(G, q)$, depending on $G$ and $q$, such that

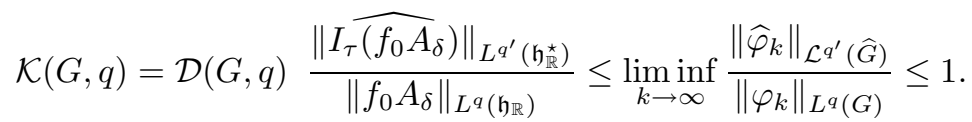

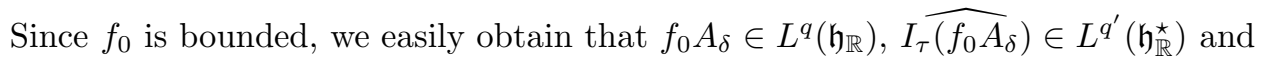
$\mathcal{K}(G, q)>0$. Therefore we have found a family $\left\{\varphi_{k}: k \geq 1\right\}$ of central functions on $G$ whose supports are eventually in $\mathcal{U}$ and such that their Hausdorff-Young quotient of exponent $q$ is bounded below by a positive constant. This concludes the proof of Theorem 5.1 for compact semisimple simply connected Lie groups.

If $G$ is not simply connected, some extra comments have to be made. We shall not give complete proofs of any of them; the details are left to the reader.

(i) Generalization (3) of formula (1) has no meaning here, but we can generalize formula (2) as

$$
\widehat{f}\left(\pi_{\lambda}\right)=\frac{1}{d_{\lambda}} \operatorname{det} W \mathcal{F}_{\mathbf{T}}\left(f B_{\delta}\right)\left(W^{t}(\lambda+\delta)-\delta\right) 1_{d_{\lambda}} .
$$

This generalization provides a couple of results parallel to Lemmas 5.2 and 5.5. Namely,

- We have $\left\{W^{t}(\lambda+\delta)-\delta: \quad W \in \mathcal{W}_{G}, \quad \lambda \in \Lambda_{\mathrm{DW}}\right\}=\Lambda_{\mathrm{W}} \backslash(\mathrm{P}-\delta)$. The mapping $(W, \lambda) \in \mathcal{W}_{G} \times \Lambda_{\mathrm{DW}} \mapsto W^{t}(\lambda+\delta)-\delta \in \Lambda_{\mathrm{W}} \backslash(\mathrm{P}-\delta)$ is injective.

- If $f: G \rightarrow \mathbb{C}$ is central, then $\mathcal{F}_{\mathfrak{h}_{\mathbb{R}}}\left(f B_{\delta}\right)(\xi)=0$ for all $\xi \in \mathrm{P}-\delta$. 
(ii) Proposition 5.3 is now replaced by the following identity, valid for central functions $f: G \rightarrow \mathbb{C}:$

$$
\|\widehat{f}\|_{\mathcal{L}^{q^{\prime}}(\widehat{G})}=\mathcal{A}(G, q)\left[\sum_{\lambda \in \Lambda_{\mathrm{W}} \backslash(\mathrm{P}-\delta)} \frac{\left|\mathcal{F}_{\mathbf{T}}\left(f B_{\delta}\right)(\lambda)\right|^{q^{\prime}}}{\prod_{\alpha \in \mathcal{R}^{+}}|\langle\alpha, \lambda+\delta\rangle|^{q^{\prime}-2}}\right]^{1 / q^{\prime}} .
$$

(iii) The bases of $\mathfrak{h}_{\mathbb{R}}^{\star}$ and $\mathfrak{h}_{\mathbb{R}}$ respectively that generate $\Lambda_{\mathrm{W}}$ and $\mathrm{L}_{\mathrm{W}}$ with integer coefficients are no longer the basis of fundamental weights and its predual. In fact, the fundamental weights generate the weight lattice of the universal covering group of $G$, which is a lattice containing $\Lambda_{\mathrm{W}}$ and strictly bigger than it. Therefore we need to define $\left\{H_{1}, H_{2}, \ldots, H_{r}\right\}$ and $\left\{\omega_{1}, \omega_{2}, \ldots, \omega_{r}\right\}$ just as the bases of $\mathfrak{h}_{\mathbb{R}}$ and $\mathfrak{h}_{\mathbb{R}}^{\star}$ respectively for which $L_{\mathrm{W}}$ and $\Lambda_{\mathrm{W}}$ have integer coefficients. Once we have clarified this point, we can define $\mathfrak{T}$ in the same way and regard $f_{0}$ as a bounded complex-valued function on $\mathfrak{h}_{\mathbb{R}}$, supported in $\mathfrak{T}$ and symmetric under the reflections that generate $\mathcal{W}_{G}$.

(iv) Let us recall that if $\delta \notin \Lambda_{\mathcal{W}}$, the function $A_{\delta}$ is not well-defined on $\mathbf{T}$. But $A_{\delta}$ is originally defined on $\mathfrak{h}_{\mathbb{R}}$, and $\delta \notin \Lambda_{\mathcal{W}}$ is not an obstacle to working with $A_{\delta}$ as a function defined on $\mathfrak{h}_{\mathbb{R}}$. On the other hand, (ii) leads us to consider, in the same spirit as in the proof given for simply connected groups, the function

$$
\widetilde{I}_{\tau}\left(f_{0} B_{\delta}\right)(\xi)=\frac{1}{\prod_{\alpha \in \mathcal{R}^{+}}|\langle\alpha, \xi+\delta\rangle|^{\tau}} \mathcal{F}_{\mathfrak{h}_{\mathbb{R}}}\left(f_{0} B_{\delta}\right)(\xi) .
$$

Now, the remark about $A_{\delta}$ shows that $\widetilde{I}_{\tau} \widehat{\left(f_{0} B_{\delta}\right)}(\xi)=\widehat{\left.I_{\tau} \widehat{\left(f_{0} A_{\delta}\right.}\right)}(\xi+\delta)$. Hence we can proceed as before, expressing the norm of this function in $L^{q^{\prime}}\left(\mathfrak{h}_{\mathbb{R}}^{\star}\right)$ as a Riemann sum, but this time we take the lattice $\Lambda_{\mathrm{W}}+\delta$ instead of $\Lambda_{\mathrm{W}}$ :

$$
\left\|\widetilde{I}_{\tau} \widehat{\left(f_{0} B_{\delta}\right)}\right\|_{L^{q^{\prime}\left(\mathfrak{h}_{\mathbb{R}}^{\star}\right)}}=\lim _{k \rightarrow \infty}\left[\sum_{\lambda \in \Lambda_{\mathrm{W}}+\delta} \frac{\mathrm{V}_{G}}{k^{r}} \frac{\left|\mathcal{F}_{\mathfrak{h}_{\mathbb{R}}}\left(f_{0} A_{\delta}\right)\left(k^{-1} \lambda\right)\right|^{q^{\prime}}}{\prod_{\alpha \in \mathcal{R}^{+}}\left|\left\langle\alpha, k^{-1} \lambda\right\rangle\right|^{\tau q^{\prime}}}\right]^{1 / q^{\prime}} .
$$

$(v)$ It is not difficult to check that $\mathcal{F}_{\mathfrak{h}_{\mathbb{R}}}\left(f_{0} A_{\delta}\right)\left(k^{-1} \lambda\right)=k^{r-\sigma} \mathcal{F}_{\mathbf{T}}\left(\varphi_{k} B_{\delta}\right)(\lambda-\delta)$, where $\varphi_{k}$ is defined as above. Hence we get

$$
\begin{aligned}
\left\|\widetilde{I}_{\tau} \widehat{\left(f_{0} B_{\delta}\right)}\right\|_{L^{q^{\prime}\left(\mathfrak{h}_{\mathbb{R}}^{\star}\right)}} & =\mathrm{V}_{G}^{1 / q^{\prime}} \lim _{k \rightarrow \infty}\left[\sum_{\lambda \in \Lambda_{\mathrm{W}} \backslash(\mathrm{P}-\delta)} \frac{\left|\mathcal{F}_{\mathbf{T}}\left(\varphi_{k} B_{\delta}\right)(\lambda)\right|^{q^{\prime}}}{\prod_{\alpha \in \mathcal{R}^{+}}|\langle\alpha, \lambda+\delta\rangle|^{\tau q^{\prime}}}\right]^{1 / q^{\prime}} \\
& =\mathcal{B}(G, q) \lim _{k \rightarrow \infty}\left\|\widehat{\varphi}_{k}\right\|_{\mathcal{L}^{q^{\prime}}(\widehat{G})} .
\end{aligned}
$$

Finally, to estimate the norm of $\varphi_{k}$ on $L^{q}(G)$, we follow the same arguments. This completes the proof of Theorem 5.1 and, consequently, the proof of Theorem 3.1

Remark 5.6. Let $\left\{\mathcal{U}_{n}: n \geq 1\right\}$ be a basis of neighborhoods of $\mathbf{1}$, and let

$$
\mathcal{K}(G, q)=\inf _{n \geq 1} \sup \left\{\frac{\|\widehat{f}\|_{\mathcal{L}^{q^{\prime}(\widehat{G})}}}{\|f\|_{L^{q}(G)}}: f \text { central, } \quad f \in L^{q}(G), \quad \operatorname{supp}(f) \subset \mathcal{U}_{n}\right\} .
$$

This constant does not depend on the chosen basis, and Theorem 5.1 states that $0<\mathcal{K}(G, q) \leq 1$ for any $1 \leq q \leq 2$ and any compact semisimple Lie group. However, it would be interesting to find the exact value of that constant. Sharp constants for 
the Hausdorff-Young inequality were investigated in [2], 3] and [13]. In the local case, if $\mathcal{B}_{q}=\sqrt{q^{1 / q} / q^{\prime 1 / q^{\prime}}}$ stands for the Babenko-Beckner constant, it is already known that $\mathcal{K}(\mathbb{T}, q)=\mathcal{B}_{q}$. Andersson proved it for $q^{\prime}$ an even integer in [1], and Sjölin completed the proof, see [15]. Also it is obvious that $\mathcal{K}(G, 1)=\mathcal{K}(G, 2)=1$ for any compact group $G$. In the general case, a detailed look at the proof of Theorem [5.1] gives that the constant $\mathcal{K}(G, q)$ is the supremum of

$$
\left|\mathcal{W}_{G}\right|^{\tau} \prod_{\alpha \in \mathcal{R}^{+}}|\langle\alpha, \delta\rangle|^{\tau} \mathrm{V}_{G}^{-1 / q^{\prime}} \lim _{k \rightarrow \infty} \frac{\left(\int_{\mathfrak{h}_{\mathbb{R}}^{+}}\left|\mathcal{F}_{\mathfrak{h}_{\mathbb{R}}}\left(f_{0} A_{\delta}(\xi)\right)\right|^{q^{\prime}} \prod_{\alpha \in \mathcal{R}^{+}}|\langle\alpha, \xi\rangle|^{2-q^{\prime}} d \xi\right)^{1 / q^{\prime}}}{\left(\int_{\mathfrak{h}_{\mathbb{R}}}\left|f_{0} A_{\delta}(x)\right|^{q}\left|k^{\left|\mathcal{R}^{+}\right|} A_{\delta}(x / k)\right|^{2-q} d x\right)^{1 / q}}
$$

for $1<q \leq 2$, where the supremum runs over the family of functions $f_{0}: \mathfrak{h}_{\mathbb{R}} \rightarrow \mathbb{C}$, supported in $\mathfrak{T}$ and symmetric under the reflections generating the Weyl group of $G$. If $\mathcal{K}_{f_{0}}(G, q)$ denotes the expression given above, then one easily gets that $\mathcal{K}_{f_{0}}(G, q)$ equals

$$
\frac{\left|\mathcal{W}_{G}\right|^{\tau}}{(2 \pi)^{\tau\left|\mathcal{R}^{+}\right|} \mathrm{V}_{G}^{1 / q^{\prime}}} \prod_{\alpha \in \mathcal{R}^{+}}|\langle\alpha, \delta\rangle|^{\tau} \frac{\left(\int_{\mathfrak{h}_{\mathbb{R}}^{\star}}\left|\mathcal{F}_{\mathfrak{h}_{\mathbb{R}}}\left(f_{0} A_{\delta}(\xi)\right)\right|^{q^{\prime}} \prod_{\alpha \in \mathcal{R}^{+}}|\langle\alpha, \xi\rangle|^{2-q^{\prime}} d \xi\right)^{1 / q^{\prime}}}{\left(\int_{\mathfrak{h}_{\mathbb{R}}}\left|f_{0} A_{\delta}(x)\right|^{q} \prod_{\alpha \in \mathcal{R}^{+}}|\langle\alpha, x\rangle|^{2-q} d x\right)^{1 / q}} .
$$

Moreover, taking $q=2$ and using the Plancherel theorem on compact groups, we see that $\mathrm{V}_{G}=1$. The boundedness of this expression can be regarded as a weighted Hausdorff-Young inequality of Pitt type; see [4] for more on this topic.

As we pointed out in the introduction, the growth of $\mathcal{C}_{q}^{1}\left(l^{p^{\prime}}(n), G\right)$ remains open for $1 \leq p<q \leq 2$. We end this paper with some remarks about this problem.

Remark 5.7. In Theorem 3.1 we found an extremal function $\Phi_{n}=\left(\varphi_{1}, \varphi_{2}, \ldots, \varphi_{n}\right)$ such that

$$
\mathcal{C}_{q}^{1}\left(l^{p}(n), G\right) \geq \frac{\left\|\widehat{\Phi}_{n}\right\|_{\mathcal{L}_{l^{\prime}(n)}^{q^{\prime}}(\widehat{G})}}{\left\|\Phi_{n}\right\|_{L_{l^{p}(n)}^{q}(G)}} \geq \mathcal{K}(G, q) n^{1 / p-1 / q} .
$$

Our functions $\varphi_{1}, \varphi_{2}, \ldots, \varphi_{n}$ satisfied two crucial properties, namely,

(P1) the norm of $\widehat{\varphi}_{k}(\pi)$ on $S_{d_{\pi}}^{q^{\prime}}$ does not depend on $k$ for any $\pi \in \widehat{G}$, and

(P2) $\varphi_{1}, \varphi_{2}, \ldots, \varphi_{n}$ have pairwise disjoint supports on $G$.

The idea was to compare the norms of $\widehat{\Phi}_{n}$ and $\Phi_{n}$ with $n^{1 / p}$ and $n^{1 / q}$ respectively. To this end, properties (P1) and (P2) were the conditions to be required, since they provided suitable simplifications for the original expressions of such norms. Now, if we replace $l^{p}(n)$ by $l^{p^{\prime}}(n)$ in the relation above, we want to compare the norms of $\widehat{\Phi}_{n}$ and $\Phi_{n}$ with $n^{1 / q^{\prime}}$ and $n^{1 / p^{\prime}}$ respectively. Notice that $1 / p-1 / q=1 / q^{\prime}-1 / p^{\prime}$. For that, we require these other properties on $\varphi_{1}, \varphi_{2}, \ldots, \varphi_{n}$ :

(P3) the absolute value $\left|\varphi_{k}(g)\right|$ does not depend on $k$ for any $g \in G$, and

(P4) $\widehat{\varphi}_{1}, \widehat{\varphi}_{2}, \ldots, \widehat{\varphi}_{n}$ have pairwise disjoint supports on $\widehat{G}$.

In the introduction we recalled that the growth of $\mathcal{C}_{q}^{1}\left(l^{p}(n), G\right)$ and $\mathcal{C}_{q}^{1}\left(l^{p^{\prime}}(n), G\right)$ can be understood as dual problems with respect to the Fourier transform operator. 
Now, these properties justify this point. Assuming properties (P3) and (P4), we get $\mathcal{C}_{q}^{1}\left(l^{p^{\prime}}(n), G\right) \geq \mathcal{K}^{\prime}(G, q, n) n^{1 / q^{\prime}-1 / p^{\prime}}$, where $\mathcal{K}^{\prime}(G, q, n)$ is given by

$$
\mathcal{K}^{\prime}(G, q, n)=\left(\frac{1}{n} \sum_{k=1}^{n}\left[\frac{\left\|\widehat{\varphi}_{k}\right\|_{\mathcal{L}^{q^{\prime}}(\widehat{G})}}{\left\|\varphi_{k}\right\|_{L^{q}(G)}}\right]^{q^{\prime}}\right)^{1 / q^{\prime}} .
$$

Hence, if we define $\mathcal{K}^{\prime}(G, q)=\inf _{n \geq 1} \mathcal{K}^{\prime}(G, q, n)$, it remains to see that $\mathcal{K}^{\prime}(G, q)>0$. We do not know if this inequality holds for any compact semisimple Lie group and any $1 \leq q \leq 2$.

Remark 5.8. We do not know if properties (P3) and (P4) are compatible. However, given $f_{0} \in L^{2}(G)$ continuous and any sequence of positive numbers $\left\{\varepsilon_{n}: n \geq 1\right\}$ decreasing to 0 , it is not difficult to see that there exists a system $\Phi=\left\{\varphi_{n}: n \geq 1\right\}$ of trigonometric polynomials on $G$ satisfying the following three conditions:

(1) The functions $\widehat{\varphi}_{1}, \widehat{\varphi}_{2}, \ldots$ have pairwise disjoint supports on $\widehat{G}$.

(2) The estimate $\left|\varphi_{n}\right| \leq\left|f_{0}\right|+\varepsilon_{n}$ holds in $G$.

(3) The estimate $\left|\varphi_{n}\right| \geq\left|f_{0}\right|-\varepsilon_{n}$ holds outside $\Omega_{n}$, where $\mu\left(\Omega_{n}\right) \rightarrow 0$ as $n \rightarrow \infty$.

Remark 5.9. As is well known, $\mathcal{C}_{q}^{1}\left(l^{p^{\prime}}(n), G\right)=n^{1 / p-1 / q}$ for any compact abelian group $G$. This equality follows by taking $\varphi_{1}, \varphi_{2}, \ldots, \varphi_{n}$ to be a collection of $n$ pairwise distinct characters. This motivates us to see what happens when we consider the irreducible characters of a compact semisimple Lie group. Let $\chi_{\lambda}$ be the character of the irreducible representation $\pi_{\lambda}$, and consider the function $\Phi_{n}(g)=\left(d_{\lambda_{1}}^{\tau} \chi_{\lambda_{1}}(g), d_{\lambda_{2}}^{\tau} \chi_{\lambda_{2}}(g), \ldots, d_{\lambda_{n}}^{\tau} \chi_{\lambda_{n}}(g)\right)$, where $\lambda_{1}, \lambda_{2}, \ldots, \lambda_{n}$ are pairwise distinct dominant weights and $\tau=1-2 / q^{\prime}$. Then we have

$$
\left\|\widehat{\Phi}_{n}\right\|_{\mathcal{L}_{l p^{\prime}(n)}^{q^{\prime}}(\widehat{G})}=\left(\sum_{k=1}^{n} d_{\lambda_{k}}\left\|d_{\lambda_{k}}^{\tau} \widehat{\chi}_{\lambda_{k}}\left(\pi_{k}\right)\right\|_{S_{d_{\lambda_{k}}}^{q^{\prime}}}^{q^{\prime}}\right)^{1 / q^{\prime}}=n^{1 / q^{\prime}} .
$$

On the other hand, applying consecutively the Weyl integration formula and the Weyl character formula, we get

$$
\left\|\Phi_{n}\right\|_{L_{p^{p^{\prime}(n)}}^{q}(G)}=\left(\frac{1}{\left|\mathcal{W}_{G}\right|} \int_{\mathbf{T}}\left(\sum_{k=1}^{n}\left|d_{\lambda_{k}} A_{\delta}(t)\right|^{\tau p^{\prime}}\left|A_{\lambda_{k}+\delta}(t)\right|^{p^{\prime}}\right)^{q / p^{\prime}} d m(t)\right)^{1 / q} .
$$

However, these relations do not provide optimal growth. For instance, in the simplest case $G=S U(2)$ it can be checked that there exists a constant $\mathcal{K}_{p, q}$, depending on $p$ and $q$, such that

$$
\frac{1}{n^{1 / p^{\prime}}}\left(\int_{S U(2)}\left\|\Phi_{n}(g)\right\|_{l^{p^{\prime}}(n)}^{q} d \mu(g)\right)^{1 / q} \geq \mathcal{K}_{p, q} n^{\tau} .
$$

Remark 5.10. If we try to find out why our attempts to get optimal growth have failed, we need to revisit the proof of Theorem 3.1 The point is that we required the functions $\varphi_{1}, \varphi_{2}, \ldots, \varphi_{n}$ not only to satisfy properties (P1) and (P2), but also to be translations of a common function. This was essential in Section 3, and here the obstacle lies in the fact that we cannot take translations, since the dual object does not have a group structure. This is the main difference from the abelian case 
where, since the dual object is a group, multiplication by a character in $G$ becomes a translation in the other side of the Fourier transform operator.

Remark 5.11. The quantized Rademacher system associated to a probability space $(\Omega, \mathcal{M}, \mu)$, an index set $\Sigma$, and a family $\left\{d_{\sigma}: \sigma \in \Sigma\right\}$ of positive integers is defined by a collection $\mathcal{R}=\left\{\rho^{\sigma}: \Omega \rightarrow O\left(d_{\sigma}\right)\right\}_{\sigma \in \Sigma}$ of independent random orthogonal matrices, uniformly distributed on the orthogonal group $O\left(d_{\sigma}\right)$. In 9 we define the notions of $\mathcal{R}$-type, $\mathcal{R}$-cotype and strong $\mathcal{R}$-cotype of an operator space $E$. Moreover, we show that

$$
\begin{array}{lll}
\text { Fourier type } p & \Rightarrow & \text { strong } \mathcal{R} \text {-cotype } p^{\prime} \\
\text { Fourier cotype } p^{\prime} & \Rightarrow \mathcal{R} \text {-type } p .
\end{array}
$$

These implications allow us to work with the quantized Rademacher system, where other techniques are available to study the growth of $\mathcal{C}_{q}^{1}\left(l^{p^{\prime}}(n), G\right)$.

Remark 5.12. Of course, the growth of $\mathcal{C}_{q}^{1}\left(l^{p^{\prime}}(n), G\right)$ is trivially optimal when we work with compact groups with infinitely many inequivalent irreducible representations of the same degree $d_{0}$. The unitary groups $U(n)$ are the simplest noncommutative examples of this degenerate case. Also, it is not difficult to check that $\mathcal{C}_{2}^{1}\left(l^{p^{\prime}}(n), G\right)=n^{1 / 2-1 / p^{\prime}}$ by the Plancherel theorem for compact groups.

\section{REFERENCES}

1. M. E. Andersson, The Hausdorff-Young inequality and Fourier type, Ph.D. Thesis, Uppsala (1993).

2. K. I. Babenko, An inequality in the theory of Fourier integrals, Izv. Akad. Nauk SSSR 25 (1961), 531 - 542; English transl., Amer. Math. Soc. Transl. (2) 44 (1965), 115 - 128. MR 25:2379

3. W. Beckner, Inequalities in Fourier analysis, Ann. of Math. (2) 102 (1975), 159 - 182. MR 52:6317

4. W. Beckner, Pitt's inequality and the uncertainty principle, Proc. Amer. Math. Soc. 123 (1995), 1897 - 1905. MR 95g:42021

5. E. G. Effros and Z. J. Ruan, Operator Spaces, London Math. Soc. Monogr. 23, Oxford Univ. Press (2000). MR 2002a:46082

6. G. B. Folland, A Course in Abstract Harmonic Analysis, Stud. Adv. Math., CRC Press (1995). MR 98c:43001

7. W. Fulton and J. Harris, Representation Theory: A First Course, Graduate Texts in Math., Springer-Verlag, New York, 1991. MR 93a:20069

8. J. García-Cuerva and J. Parcet, Vector-valued Hausdorff-Young inequality on compact groups, Submitted for publication.

9. J. García-Cuerva and J. Parcet, Quantized orthonormal systems: A non-commutative Kwapień theorem, Studia Math. 155 (2003), 273 - 294.

10. R. A. Kunze, $L_{p}$ Fourier transforms on locally compact unimodular groups, Trans. Amer. Math. Soc. 89 (1958), 519 - 540. MR 20:6668

11. G. Pisier, The Operator Hilbert Space OH, Complex Interpolation and Tensor Norms, Mem. Amer. Math. Soc. 122 (1996), No. 585. MR 97a:46024

12. G. Pisier, Non-commutative vector valued $L_{p}$-spaces and completely p-summing maps, Astérisque (Soc. Math. France) 247 (1998). MR 2000a:46108

13. B. Russo, The norm of the $L^{p}$-Fourier transform on unimodular groups, Trans. Amer. Math. Soc. 192 (1974), 293 - 305. MR 55:8689a 
14. B. Simon, Representations of Finite and Compact Groups, Graduate Stud. Math. 10, Amer. Math. Soc., Providence, RI, 1996. MR 97c:22001

15. P. Sjölin, A remark on the Hausdorff-Young inequality, Proc. Amer. Math. Soc. 123 (1995), 3085 - 3088. MR 95m:42007

Department of Mathematics, Universidad Autónoma de Madrid, Madrid 28049, Spain

E-mail address: jose.garcia-cuerva@uam.es

Department of Mathematics, Universidad Autónoma de Madrid, Madrid 28049, Spain

Department of Mathematics, Universidad Autónoma de Madrid, Madrid 28049, Spain

E-mail address: javier.parcet@uam.es 